\title{
Hole-transporting Poly(dendrimer)s as Electron Donors for Low Donor Organic Solar Cells with Efficient Charge Transport
}

\author{
Wei Jiang ${ }^{1 \S}$, Martin Stolterfoht ${ }^{2 \S}$, Hui Jin ${ }^{1}$, and Paul L. Burn ${ }^{1 *}$ \\ ${ }^{1}$ Centre for Organic Photonics \& Electronics (COPE), School of Chemistry and Molecular Biosciences, The \\ University of Queensland, St. Lucia, QLD 4072, Australia \\ ${ }^{2}$ Institute of Physics and Astronomy, University of Potsdam, Karl-Liebknecht-Str. 24-25, D-14476 Potsdam-Golm, \\ Germany \\ sthese authors contributed equally to this work \\ *E-mail: p.burn2@uq.edu.au
}



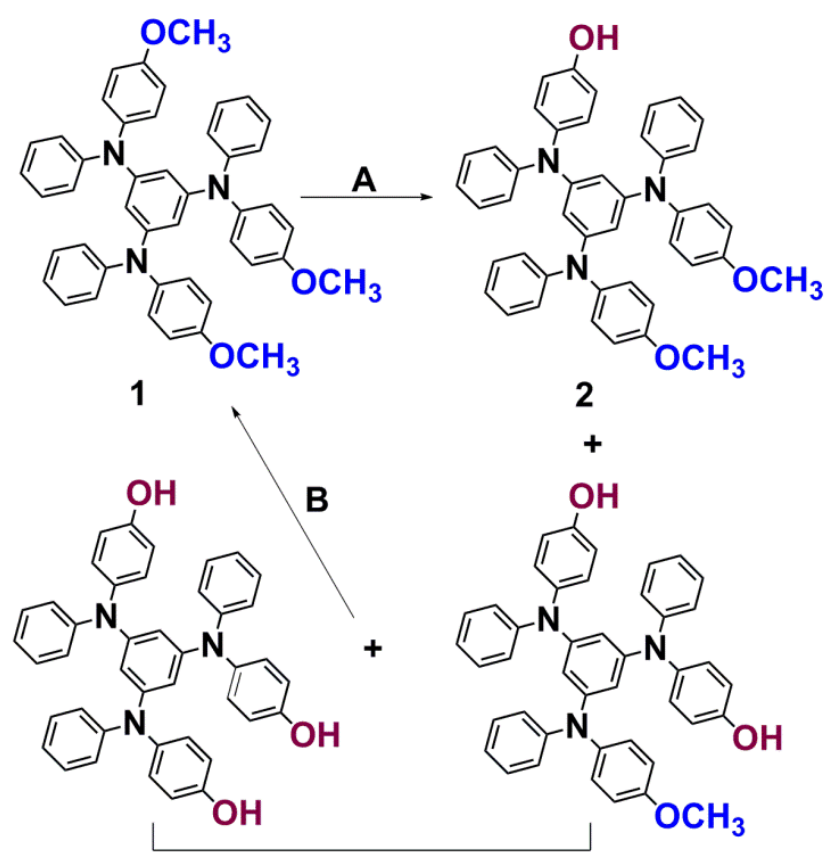

di- and tri-demethylate byproducts

A: Aluminum chloride, sodium iodide, toluene, $60{ }^{\circ} \mathrm{C}, 27 \%$; B: Dimethyl carbonate, tetra- $n$-butylammonium bromide, potassium carbonate, reflux, $57 \%$.

Figure S1 Strategy for recovering dendrimer 1 from the di- and tri-demethylated side products. 


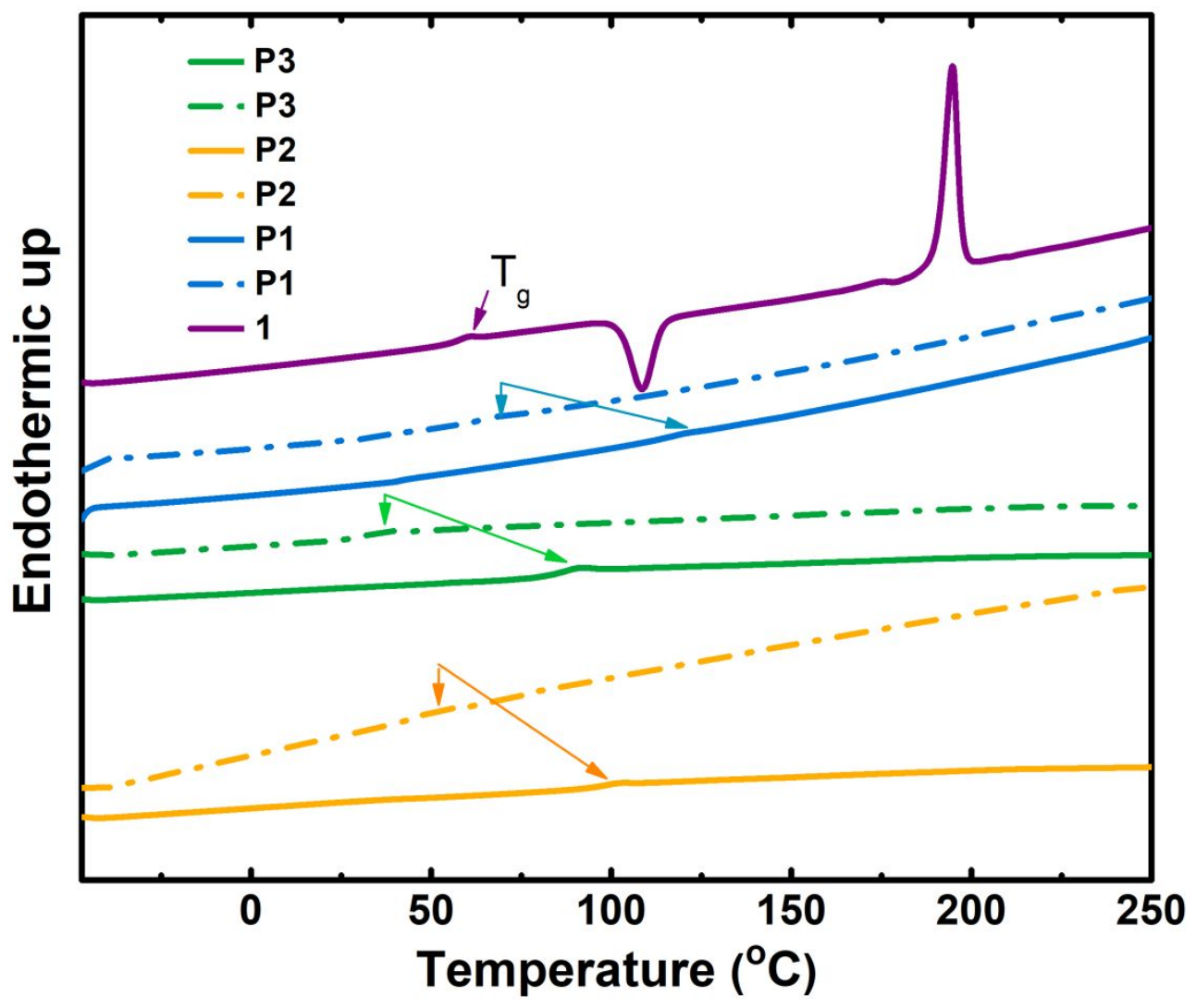

Figure S2 DSC plots for the three poly(dendrimer)s (solid lines) in comparison with the corresponding monomers (dash-dot lines) and that of dendrimer 1. The plots shown are for the second heating process at scan rate of $50{ }^{\circ} \mathrm{C} / \mathrm{min}$. 


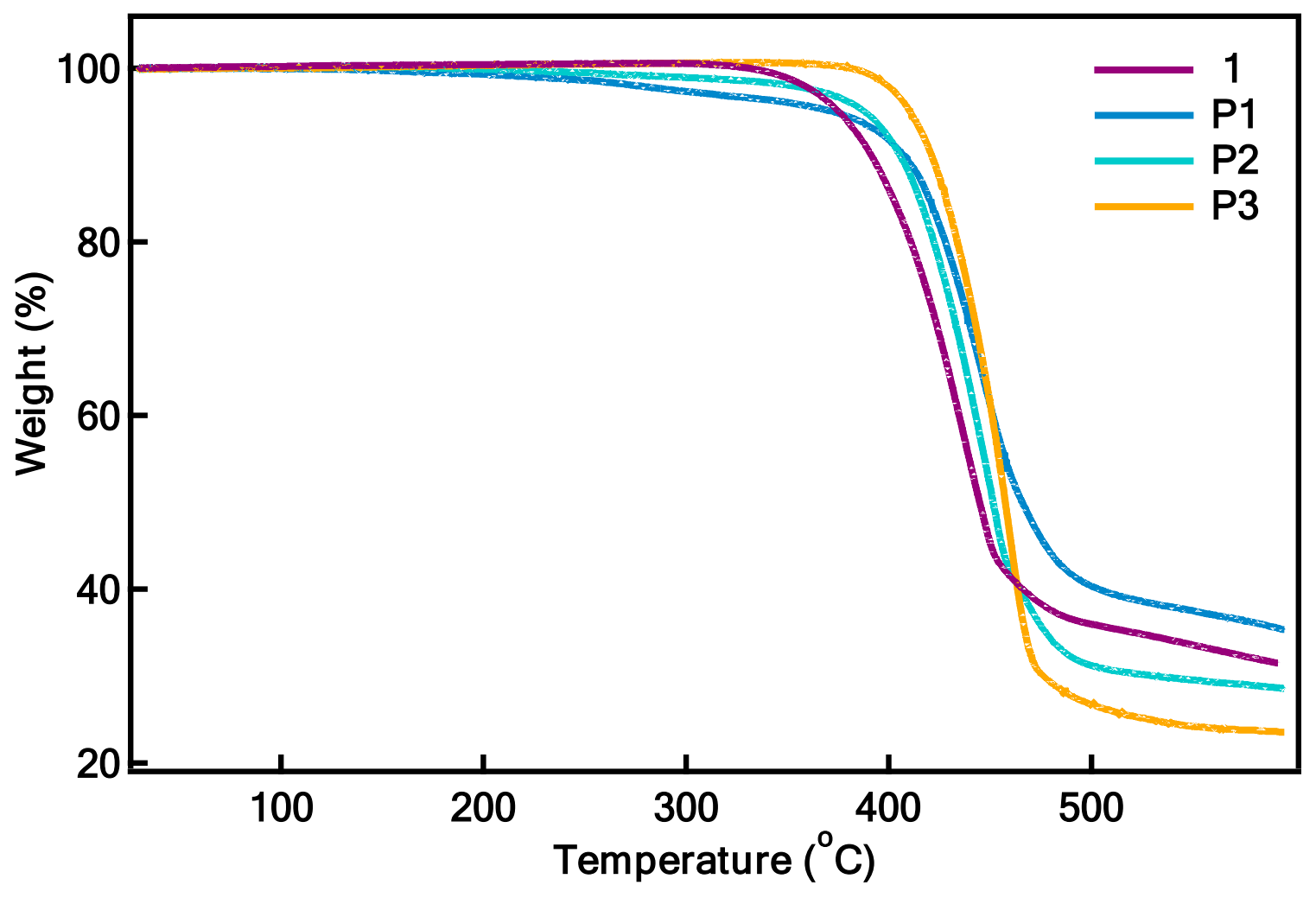

Figure S3 TGA plots for 1 and the poly(dendrimer)s.

(a) 


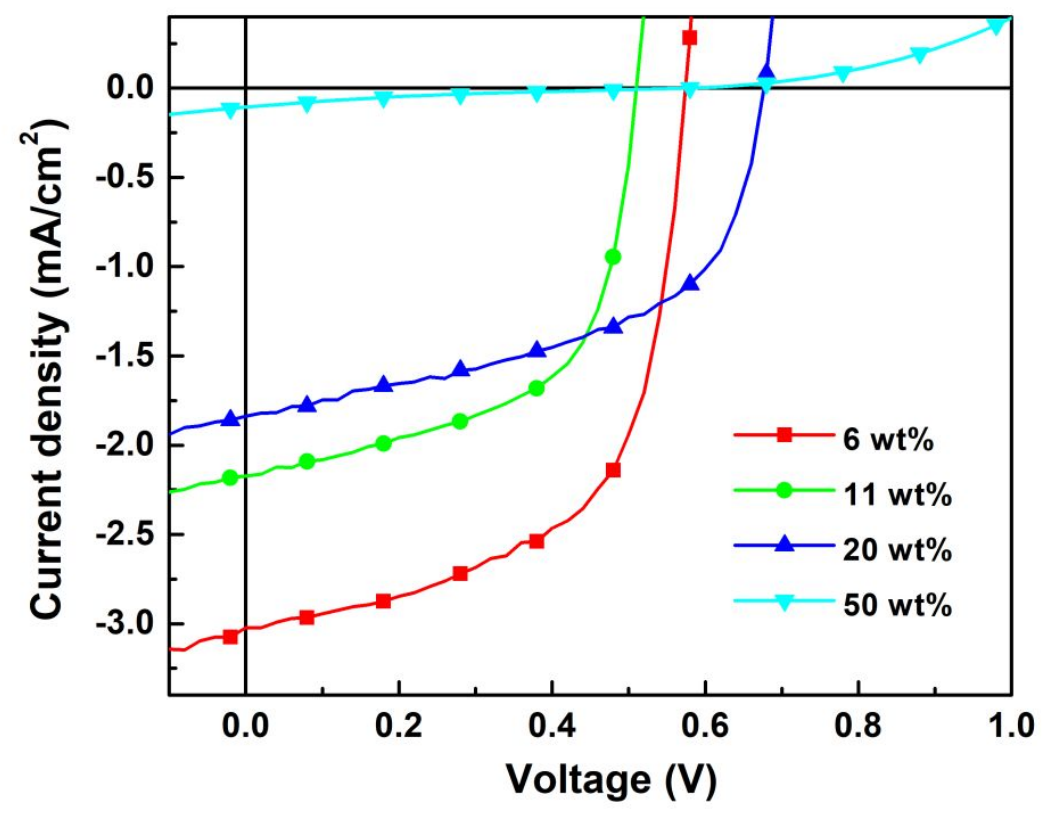

(b)

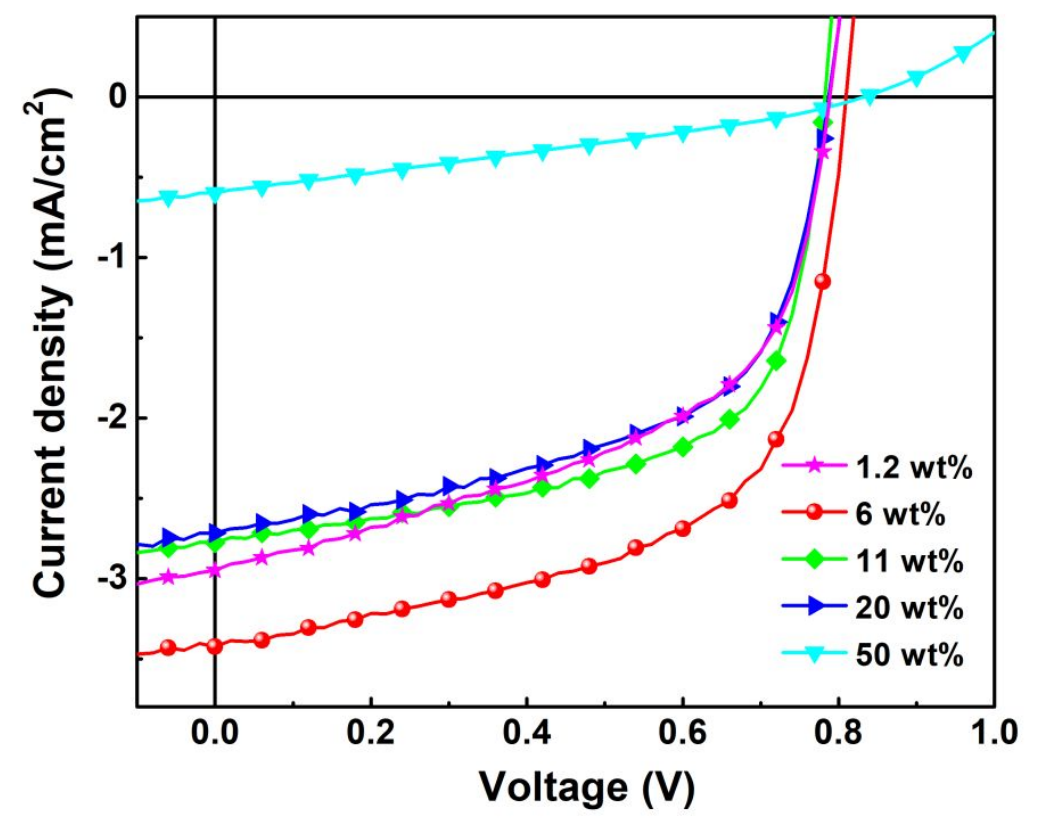

(c) 


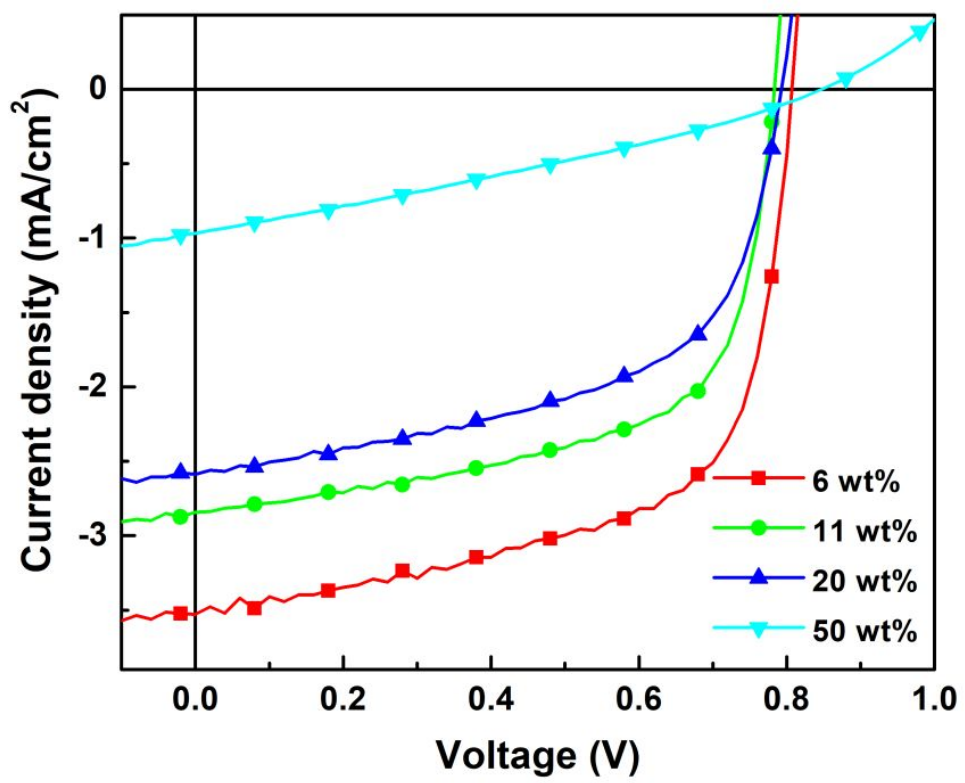

Figure S4 Current density-voltage ( $J-V$ ) curves of $\mathrm{BHJ}$ devices with a) $\left.\mathbf{P 1}: \mathrm{PC}_{70} \mathrm{BM}, \mathrm{b}\right)$ P2:PC ${ }_{70} \mathrm{BM}$, and c) P3: $\mathrm{PC}_{70} \mathrm{BM}$ of different poly(dendrimer) blend concentrations.

Table S1 Averaged device performance parameters from at least 4 cells for P1:PC ${ }_{70} \mathrm{BM}$ (a), P2:PC ${ }_{70} \mathrm{BM}$ (b), and P3:PC ${ }_{70} \mathrm{BM}$ (c) of different poly(dendrimer) blend concentrations. Among devices with different polymer content, the device with $6 \mathrm{wt} \%$ performed the best with a high $J_{s c}$ and $F F$. By increasing the donor content up to $50 \mathrm{wt} \%$, the reduction in photocurrent generation is responsible for the substantial decrease of the $J_{\mathrm{sc}}$, which at least in part due to the fact that the poly(dendrimer) does not absorb strongly at visible wavelengths. In addition, for the low donor content films, the balanced electron-hole transport results in the high $F F$ due to the suppressed bimolecular recombination.

(a)

$\begin{array}{ccccc}\begin{array}{c}\text { Blends } \\ {\left[\mathbf{P 1}(\mathbf{w t} \%): \mathbf{P C}_{\mathbf{7 0}} \mathbf{B M}\right]}\end{array} & \begin{array}{c}\boldsymbol{J}_{\boldsymbol{s c}} \\ \left(\mathbf{m A} / \mathbf{c m}^{2}\right)\end{array} & \begin{array}{c}\boldsymbol{V}_{\text {oc }} \\ (\mathbf{V})\end{array} & \boldsymbol{F F} & \begin{array}{c}\boldsymbol{P C E} \\ (\mathbf{\%})\end{array} \\ 6 & 2.94 \pm 0.07 & 0.57 \pm 0.01 & 0.60 \pm 0.01 & 1.00 \pm 0.03 \\ 11 & 2.05 \pm 0.08 & 0.49 \pm 0.01 & 0.59 \pm 0.01 & 0.60 \pm 0.04 \\ 20 & 1.82 \pm 0.02 & 0.67 \pm 0.01 & 0.53 \pm 0.01 & 0.65 \pm 0.01\end{array}$


(b)

$\begin{array}{ccccc}\begin{array}{c}\text { Blends } \\ {\left[\mathbf{P 2}(\mathbf{w t} \%): \mathbf{P C}_{\mathbf{7 0}} \mathbf{B M}\right]}\end{array} & \begin{array}{c}\boldsymbol{J}_{\boldsymbol{s c}} \\ \left(\mathbf{m A} / \mathbf{c m}^{2}\right)\end{array} & \begin{array}{c}\boldsymbol{V}_{\text {oc }} \\ (\mathbf{V})\end{array} & \boldsymbol{F F} & \begin{array}{c}\boldsymbol{P C E} \\ (\%)\end{array} \\ 1.2 & 2.92 \pm 0.08 & 0.78 \pm 0.01 & 0.51 \pm 0.01 & 1.16 \pm 0.06 \\ 6 & 3.41 \pm 0.06 & 0.79 \pm 0.01 & 0.60 \pm 0.01 & 1.63 \pm 0.04 \\ 11 & 2.67 \pm 0.07 & 0.77 \pm 0.01 & 0.63 \pm 0.01 & 1.29 \pm 0.05 \\ 20 & 2.61 \pm 0.08 & 0.77 \pm 0.01 & 0.56 \pm 0.01 & 1.14 \pm 0.07 \\ 50 & 0.56 \pm 0.05 & 0.84 \pm 0.02 & 0.29 \pm 0.00 & 0.13 \pm 0.01\end{array}$

(c)

$\begin{array}{ccccc}\begin{array}{c}\text { Blends } \\ {\left[\mathbf{P 3}(\mathbf{w t} \%): \mathbf{C C}_{\mathbf{7 0}} \mathbf{B M}\right]}\end{array} & \begin{array}{c}\boldsymbol{J}_{\boldsymbol{s c}} \\ \left(\mathbf{m A} / \mathbf{c m}^{2}\right)\end{array} & \begin{array}{c}\boldsymbol{V}_{\text {oc }} \\ (\mathbf{V})\end{array} & \boldsymbol{F F} & \begin{array}{c}\boldsymbol{P C E} \\ (\%)\end{array} \\ 6 & 3.50 \pm 0.03 & 0.79 \pm 0.01 & 0.63 \pm 0.00 & 1.74 \pm 0.03 \\ 11 & 2.64 \pm 0.14 & 0.77 \pm 0.01 & 0.62 \pm 0.01 & 1.26 \pm 0.09 \\ 20 & 2.53 \pm 0.07 & 0.78 \pm 0.00 & 0.57 \pm 0.01 & 1.12 \pm 0.04 \\ 50 & 0.91 \pm 0.06 & 0.85 \pm 0.01 & 0.29 \pm 0.01 & 0.22 \pm 0.02\end{array}$


(a)

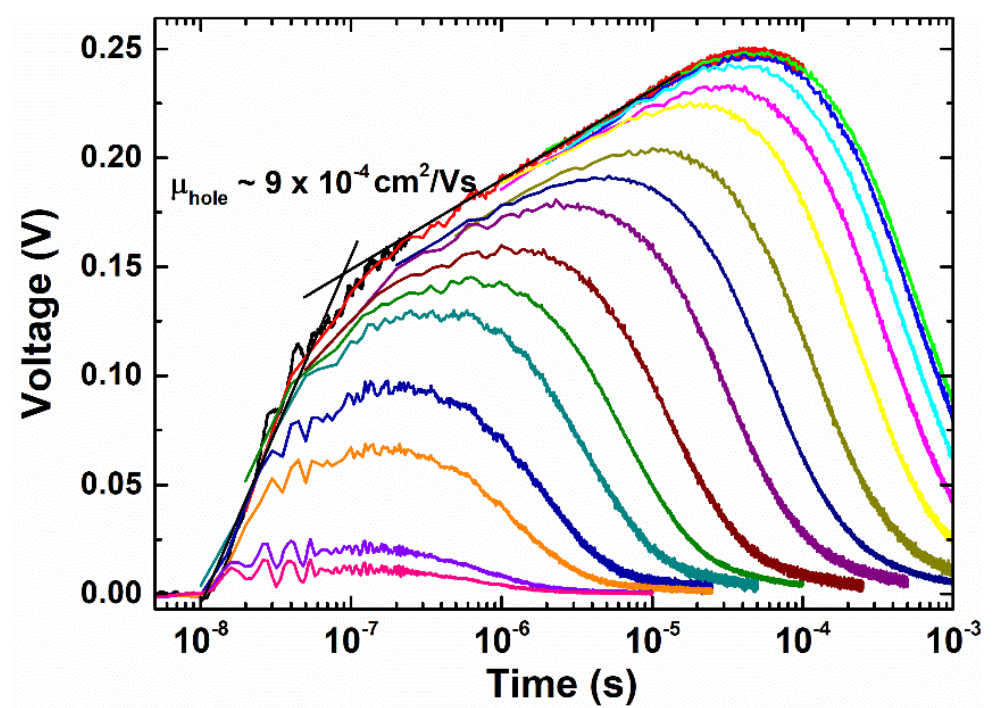

(b)

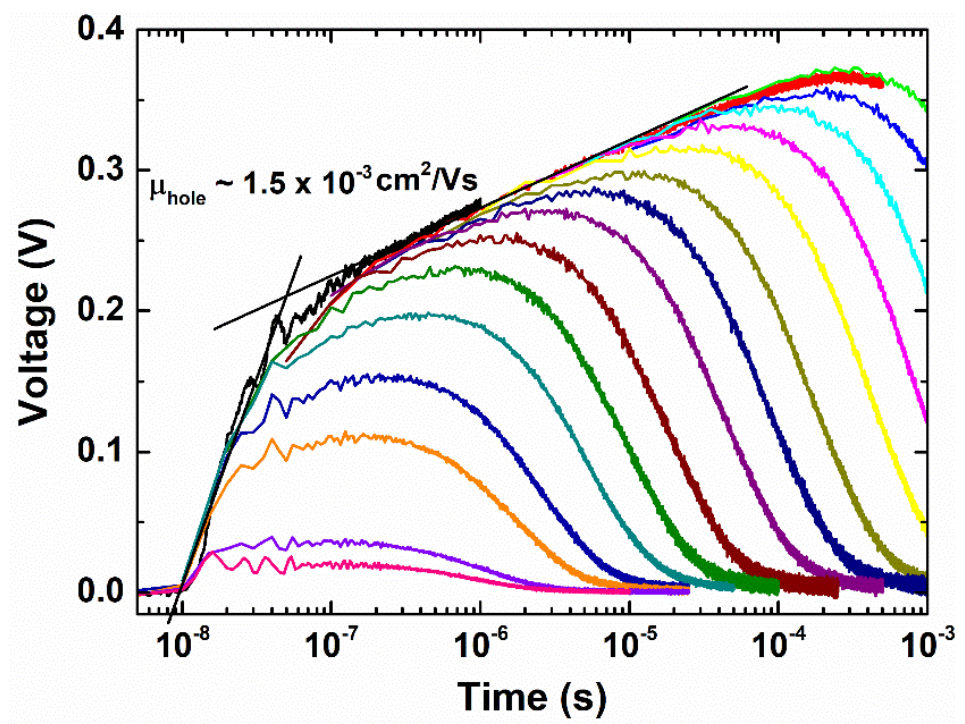

Figure S5 Resistance dependent PhotoVoltage (RPV) transient measurements on films of neat (a) dendrimer $\mathbf{1}$ and (b) and P2. 


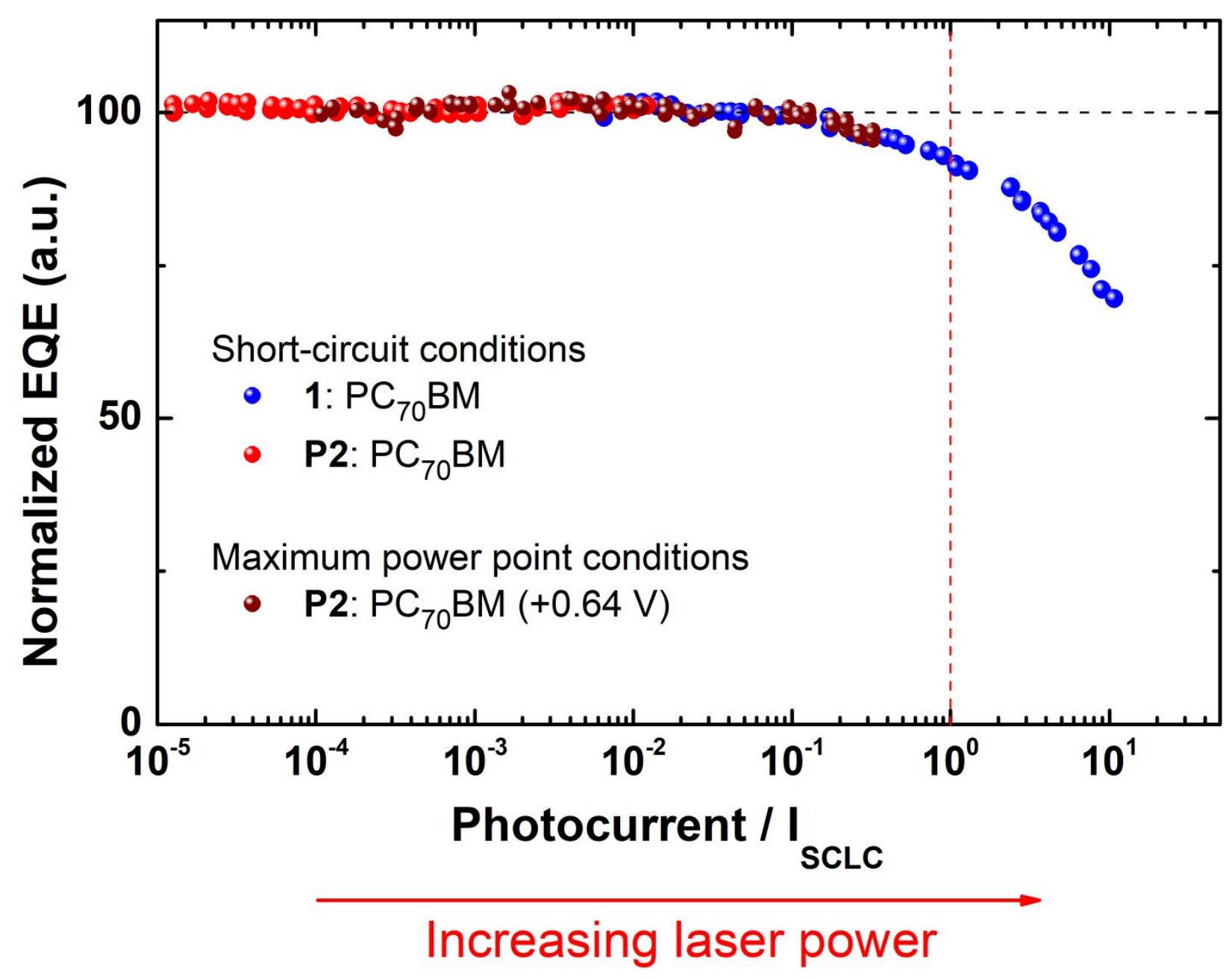

Figure S6 EQEs as a function of the photocurrent normalized to the space-charge-limited photocurrent $\left(I_{\mathrm{SCLC}}\right)$ for the dendrimer 1:PC $\mathrm{PC}_{70} \mathrm{BM}$ blend at short-circuit conditions as well as for the P2: $\mathrm{PC}_{70} \mathrm{BM}$ blend at short-circuit and maximum power point. The $\mathrm{EQE}$ was obtained from the intensity dependent photocurrent (IPC) measurements as described in the main text and the $I_{\text {SCLC }}$ was calculated according to previously reported work from the slower carrier mobility (Figure 4 in the main text) by assuming a Langevin recombination coefficient. ${ }^{1}$ Ref. 1 demonstrated how the charge transport parameters determine the bimolecular recombination losses that manifest in the deviation from a constant $\mathrm{EQE} v$ s. photocurrent or laser power. The figure shows that the $I_{\mathrm{SCLC}}$, as predicted from the slower carrier mobility obtained from RPV, coincides with the experimentally measured EQE deviation. This confirms that the hole mobilities as obtained by RPV are relevant for understanding the device performance. Note, we could not reach the $I_{\mathrm{SCLC}}$ with the available laser power for the $\mathbf{P 2}: \mathrm{PC}_{70} \mathrm{BM}$ blend device at short-circuit conditions due to the relatively thin active layer and high hole mobility. We note that the intensity was increased until $\sim 3$ equivalent 
suns, which implies that the linear photocurrent would be expected to continue until much higher intensities ( $>30$ suns). Therefore, we applied a forward bias of $0.64 \mathrm{~V}$ (corresponding to the voltage at the maximum power point) to increase the carrier transit time and decrease the critical $I_{\mathrm{SCLC}}$.

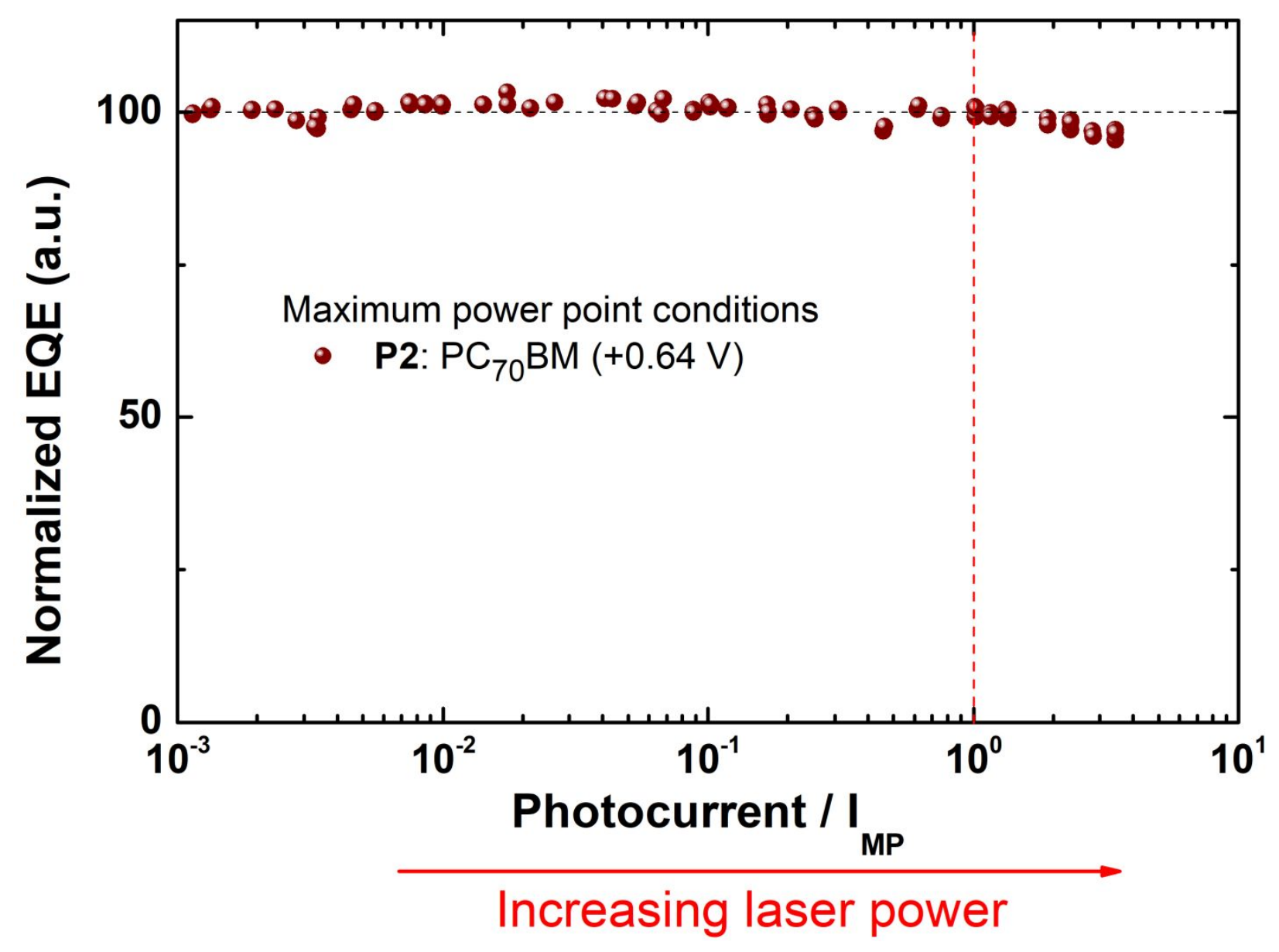

Figure $\mathbf{S 7} \mathrm{EQE}$ as a function of the photocurrent normalized to the photocurrent at the maximum power point $\left(I_{\mathrm{MP}}\right)$ in P2:PC $\mathrm{P}_{70} \mathrm{BM}$ blend at maximum power point conditions $\left(V_{\mathrm{MP}}\right)$. The flat $\mathrm{EQE}$ demonstrates the absence of second-order bimolecular recombination losses even at $V_{\mathrm{MP}}$ due to the high hole mobilities and the relatively thin active layer, giving rise to the high $F F$ of 0.65 .

\section{REFERENCE}

1. Stolterfoht, M.; Armin, A.; Philippa, B.; White, R. D.; Burn, P. L.; Meredith, P.; Juška, G.; Pivrikas, A., Photocarrier drift distance in organic solar cells and photodetectors. Scientific Reports 2015, 5, 9949. 